\title{
Thermodynamic Limit of Correlation Functions in a System of Gravitating Fermions
}

\author{
Bernhard Baumgartner \\ Institut für theoretische Physik der Universität Wien, Wien, Austria
}

\begin{abstract}
We show that the correlation functions in a system of gravitating fermions converge as tempered distributions in the thermodynamic limit, if the system is not at the point of phase-transition. The densities converge to the density of the Thomas-Fermi-theory and are not correlated in the limit.
\end{abstract}

\section{Introduction}

It has been shown by $\mathrm{P}$. Hertel et al. $([1,2])$ that non-relativistic gravitating fermions have a kind of thermodynamic limit and that in the limit the system is governed by temperature-dependent Thomas-Fermi- (T.F.-) equations. What is unusual in this limit is the dependence of parameters on the particle-number $N$ : the system is confined to a region, the linear dimensions of which vary as $N^{-1 / 3}$ and the temperature is set proportional to $N^{4 / 3}$ or the energy proportional to $N^{7 / 3}$, if one works with the microcanonical ensemble.) The free energy divided by $N^{7 / 3}$ has then a definite finite limit when $N$ tends to infinity. To make things conceptually simpler and to obtain a certain similarity to the usual thermodynamic formulas, we transform the Hamiltonian

$$
H_{N}=\sum_{i=1}^{N} p_{i}^{2} / 2 m-\kappa \sum_{i<j}\left|x_{i}-x_{j}\right|^{-1}
$$

with the unitary transformation

$$
x \mapsto N^{-1 / 3} x, p \mapsto N^{1 / 3} p
$$

and divide it by $N^{4 / 3}$.

The resulting Hamiltonian,

$$
\tilde{H}_{N}=N^{-2 / 3} \sum_{i=1}^{N} p_{i}^{2} / 2 m-\kappa / N \sum_{i<j}\left|x_{i}-x_{j}\right|^{-1}
$$

with Dirichlet boundary-conditions in $L^{2}(V),(V$ does not depend on $N)$ serves to define a canonical ensemble with temperature $\beta^{-1}$ (which is also $N$-independent). 
We thus obtain a sequence of ensembles, for which there exists the "thermodynamic" limit of the free energy per particle

$$
f(N, \beta)=-(\beta N)^{-1} \log \operatorname{Tr} \exp \left(-\beta \tilde{H}_{N}\right) .
$$

In this paper we investigate the corresponding thermodynamic limit of the local density and correlation-functions.

\section{The Strategy}

The density at a point $\varrho(x)$ cannot be obtained as an expectation value of an operator, it is only a quadratic form [3]. Therefore, we shall deal with integrated densities

$$
\varrho(w)=\int d^{3} x \varrho(x) w(x), w \text { is any test-function in } \mathscr{S}\left(R^{3}\right) .
$$

We normalize the density so that its integral over all space equals 1 . In a system with $N$ particles, (5) is then the expectation value of the operator

$$
\hat{\varrho}_{N}(w)=N^{-1} \sum_{i=1}^{N} w\left(x_{i}\right) .
$$

With the help of the symmetrized product of $n$ test functions

$$
S\left(x_{1} \ldots x_{n}\right)=n !^{-1} \sum_{\text {Permutations } p(\alpha)} \prod_{\alpha=1}^{n} w_{\alpha}\left(x_{p(\alpha)}\right)
$$

we define the operator for integrated correlation functions:

$$
\hat{\varrho}_{N}\left(w_{1} \ldots w_{n}\right)=N^{-n} \sum_{i_{1} \ldots i_{n}} S\left(x_{i_{1}} \ldots x_{i_{n}}\right) \text {. }
$$

All operators of the form (6) or (8) are bounded. Their equilibrium values can therefore be written as derivatives of concave functions $([4,5])$ :

$$
\begin{aligned}
& \left\langle\hat{\varrho}_{N}\left(w_{1} \ldots w_{n}\right)\right\rangle_{\beta}=\operatorname{Tr}\left\{\hat{\varrho}_{N}\left(w_{1} \ldots w_{n}\right) \exp \left(-\beta \tilde{H}_{N}\right)\right\} \exp \beta N f(N, \beta, 0) \\
& =\left.\frac{\partial}{\partial \lambda} f(N, \beta, \lambda)\right|_{\lambda=0} \\
& f(N, \beta, \lambda)=-(\beta N)^{-1} \log \operatorname{Tr} \exp \left[-\beta\left(\tilde{H}_{N}+\lambda N \hat{\varrho}_{N}\left(w_{1} \ldots w_{n}\right)\right)\right] \text {. }
\end{aligned}
$$

The basic concept we shall follow is to show that the thermodynamic limit exists for $f(N, \beta, \lambda)$ and the limit of derivatives equals the derivative of the limiting function $\phi(\beta, \lambda)$ (which is also concave in $\lambda$ ).

\section{The Thomas-Fermi-Equations}

Following [1], we have to approximate $V(x, y)=\kappa /|x-y|$ by $V_{s}(x, y)=$ $\kappa(1-\exp (-s|x-y|) /|x-y|$, then we have to divide the Volume $V$ into cells $\Lambda_{a}(a=1 \ldots g)$ and to approximate again $V_{s}(x, y)$ from below by potentials $V_{s, g}(x, y)$, 
which are constant in each pair of cells. In the same manner we define $S_{s, g}\left(x_{1} \ldots x_{n}\right)$ to be step functions, which are constant in each $n$-tuple of cells and approximate $S\left(x_{1} \ldots x_{n}\right) . S_{s}$ can be taken equal to $S$.

In analogy to (3) and (4) we define:

$$
\begin{aligned}
H_{N, \sigma}(\lambda) & =N^{-2 / 3} \sum p_{i}^{2} / 2 m-\frac{1}{N} \sum_{i<j} V_{\sigma}\left(x_{i}, x_{j}\right)+\lambda N^{-n+1} \sum_{i_{1} \ldots i_{n}} S_{\sigma}\left(x_{i_{1}} \ldots x_{i_{n}}\right), \\
f_{\sigma}(N, \beta, \lambda) & =-(\beta N)^{-1} \log \operatorname{Tr} \exp \left[-\beta H_{N, \sigma}(\lambda)\right] .
\end{aligned}
$$

The index $\sigma$ stands for either $s, g$ or $s$ or none.

Without complications, the results of [1] for $\lambda=0$ may be generalized:

$$
\begin{aligned}
& \exists \lim _{N \rightarrow \infty} f_{s, g}(N, \beta, \lambda)=\phi_{s, g}(\beta, \lambda), \\
& \exists \lim _{N \rightarrow \infty} f(N, \beta, \lambda)=\lim _{s \rightarrow \infty} \lim _{g \rightarrow \infty} \phi_{s, g}(\beta, \lambda) .
\end{aligned}
$$

$\phi_{s, g}$ is the free energy of the T.F.-theory:

$$
\begin{aligned}
& \phi_{\sigma}=\mu_{\sigma}+\int_{V} d^{3} x \varrho_{\sigma}(x)\left[\frac{1}{2} U_{\sigma}(x)-\lambda(n-1) / n S_{\sigma}(x)\right] \\
& \quad-\beta^{-1} \int_{V} d^{3} x \int d^{3} p(2 \pi)^{-3} \log \left\{1+\exp \left[-\beta\left(p^{2} / 2 m-\mu_{\sigma}(x)\right)\right]\right\}, \\
& U_{\sigma}(x)=\int_{V} V_{\sigma}(x, y) \varrho_{\sigma}(y) d^{3} y, \\
& S_{\sigma}(x)=n \int_{V^{n-1}} S_{\sigma}\left(x_{1} x_{2} \ldots x_{n}\right) \prod_{i=2}^{n} \varrho_{\sigma}\left(x_{i}\right) d^{3} x_{i} .
\end{aligned}
$$

The $\varrho$ 's and $\mu$ 's are solutions of T.F.-equations:

$$
\begin{aligned}
& \mu_{\sigma}(x)=\mu_{\sigma}+U_{\sigma}(x)-\lambda S_{\sigma}(x), \\
& \varrho_{\sigma}(x)=\int d^{3} p(2 \pi)^{-3}\left\{1+\exp \left[\beta\left(p^{2} / 2 m-\mu_{\sigma}(x)\right)\right]\right\}^{-1} .
\end{aligned}
$$

The value of the constant $\mu_{\sigma}$ has to be chosen such that

$$
\int_{V} \varrho_{\sigma}(x) d^{3} x=1
$$

Consider:

$$
\begin{aligned}
& \Psi_{\sigma}(\varrho, \beta, \lambda)=-\frac{1}{2} \int_{V \times V} d^{3} x d^{3} y \varrho(x) V_{\sigma}(x, y) \varrho(y)+\lambda \int_{V^{n}} S_{\sigma}\left(x_{1} \ldots x_{n}\right) \prod_{i=1}^{n} \varrho\left(x_{i}\right) d^{3} x_{i} \\
& +\int_{V} d^{3} x \varrho(x) \mu(x)-\beta^{-1} \int_{V} d^{3} x \int d^{3} p(2 \pi)^{-3} \log \left\{1+\exp \left[-\beta\left(p^{2} / 2 m-\mu(x)\right)\right]\right\} .
\end{aligned}
$$

$\mu(x)$ is here a function of $\varrho(x)$, implicitly defined by Equation (19). One verifies easily the validity of the variational principle:

$$
\begin{aligned}
\phi_{\sigma}(\beta, \lambda) & =\min _{\varrho \in \Omega} \Psi_{\sigma}(\varrho, \beta, \lambda), \\
\Omega & =\left\{\varrho \in L^{1}(V): \varrho(x) \geqq 0, \int_{V} \varrho(x) d^{3} x=1\right\} .
\end{aligned}
$$




\section{Behavior of the T.F.-Theory When $g$ and $s$ Tend to Infinity}

Our aim is to show that $\phi(\beta, \lambda)=\lim _{s \rightarrow \infty} \lim _{g \rightarrow \infty} \phi_{s, g}(\beta, \lambda)$. The proof follows [2], but we have to be careful about technicalities, since rotational invariance is broken by $S_{\sigma}\left(x_{1} \ldots x_{n}\right)$.

As functions of $x$, all $V_{\sigma}(x, y)$ are in a uniformly bounded set in $L^{p}(V)(p<3)$ :

$$
\left\|V_{s, g}(x, y)\right\|_{p} \leqq\left\|V_{s}(x, y)\right\|_{p} \leqq\left\|\kappa|x-y|^{-1}\right\|_{p} \leqq\left\|\kappa|x|^{-1}\right\|_{p}=: c_{p} \text {. }
$$

The $U_{\sigma}(x)$ are convex combinations of the $V_{\sigma}(x, y)$; hence, its $p$-norms are also bounded by $c_{p}$. The $S_{\sigma}$ 's are bounded in all $L^{p}$ 's by some constant $k$.

It is more difficult to find a bound to the set of constants $\mu_{\sigma}$. We study the function

$$
g(\beta, \mu)=\int d^{3} p(2 \pi)^{-3}\left(1+\exp \beta\left(p^{2}-\mu\right)\right)^{-1} .
$$

With this function, (19) can be written as $\varrho_{\sigma}(x)=(2 m)^{3 / 2} g\left(\beta, \mu_{\sigma}(x)\right) . g(\beta, \mu)$ has the properties:

$$
\begin{aligned}
0<\frac{\partial}{\partial \mu} g(\beta, \mu) \leqq & \beta g(\beta, \mu) \quad(\text { monotonicity in } \mu), \\
g(\beta, \mu)= & \beta^{-3 / 2} g(1, \beta \mu) \leqq \theta(1-\beta \mu) \beta^{-3 / 2} g(1,1)+\theta(\beta \mu-1)\left(6 \pi^{2}\right)^{-1}\left(\mu^{3 / 2}\right. \\
& \left.+\frac{3}{2} \mu \beta^{-3 / 2}+\frac{3}{2} \beta^{-3 / 2}\right) .
\end{aligned}
$$

Because of the $L^{p}$-boundedness of the potentials, there exists a number $b$ such that

$$
\int_{V} d^{3} x \theta\left(\left|\mu_{\sigma}(x)-\mu_{\sigma}\right|-b\right)<v / 2\left(v=\int_{V} d^{3} x\right) .
$$

And with $A_{\sigma}=\left\{x: \varrho_{\sigma}(x)>(2 m)^{3 / 2} g\left(\beta, \mu_{\sigma}-b\right)\right\}$, this implies

$$
\int_{V-A_{\sigma}} d^{3} x<v / 2 \text {. }
$$

We can define $\hat{\mu}$ by $\frac{1}{2} v(2 m)^{3 / 2} g(\beta, \hat{\mu}-b)=1$, and the inequality $\mu_{\sigma}<\hat{\mu}$ follows from the monotonicity (24) and

$$
1=\int_{V} \varrho_{\sigma}(x) d^{3} x>\int_{A_{\sigma}} \varrho_{\sigma}(x) d^{3} x>\frac{1}{2} v(2 m)^{3 / 2} g\left(\beta, \mu_{\sigma}-b\right) .
$$

Up to now we know that all $\mu_{\sigma}(x)$ form a bounded set in each $L^{p}$ if $p<3$. (25) tells us that $\varrho(\mu)$ does not grow stronger than $\mu^{3 / 2}$ so that the $\varrho_{\sigma}(x)$ are in a bounded set in every $L^{2 p / 3}$ :

$$
q<2 \Rightarrow \exists d_{q}:\left\|\varrho_{\sigma}(x)\right\|_{q} \leqq d_{q} .
$$

Now we use Hölders inequality:

$$
\max _{x \in V}\left|\int V_{\sigma}(x, y) \varrho_{\sigma}(y) d^{3} y\right| \leqq \max _{x \in V}\left\|V_{\sigma}(x, y)\right\|_{p}\left\|\varrho_{\sigma}(y)\right\|_{q} \leqq c_{p} d_{q},
$$

where the following relations must hold:

$$
2<p<3,3 / 2<q<2,1 / p+1 / q=1 .
$$


This gives a uniform $L^{\infty}$-bound for all densities:

$$
\left|\varrho_{\sigma}(x)\right| \leqq(2 m)^{3 / 2} g\left(\beta, \hat{\mu}+c_{p} d_{q}+k\right) \text {. }
$$

To be able to make statements about convergence, we define, in addition to $U_{s, g}(x)$, functions $W_{s, g}(x)=\int_{V} d^{3} y V_{s}(x, y) \varrho_{s, g}(y)$ and

$$
T_{s, g}(x)=n \int_{V^{n-1}} S\left(x_{1} x_{2} \ldots x_{n}\right) \prod_{i=2}^{n} \varrho_{s, g}\left(x_{i}\right) d^{3} x_{i}
$$

which are uniformly continuous:

$$
\left|\vec{\nabla} W_{s, g}(\vec{x})\right|=\left|\int_{V} d^{3} y \varrho_{s, g}(y) \nabla_{x} V_{s}(x, y)\right| \leqq\left\|\varrho_{s, g}\right\|_{\infty} \int_{V}|\nabla(1 /|x|)| d^{3} x=\text { const }<\infty .
$$

They therefore form two conditionally compact sets (theorem of Ascoli), and have pointwise converging subsequences as $g \rightarrow \infty$, which is also true for the $U_{s, g}$, since

$$
\lim _{g \rightarrow \infty}\left|U_{s, g}(x)-W_{s, g}(x)\right|=0,
$$

and equally for the $S_{s, g}$.

The same applies to the limit $s \rightarrow \infty$. The densities also have a converging subsequence (say to $\varrho$ ) since they are well behaved functions of the $U_{\sigma}(x)$ and $S_{\sigma}(x)$.

To see that the limits are again solutions of T.F.-equations we observe that the $\Psi_{\sigma}$ 's are continuous as functionals over $L^{1} \cap L^{\infty}$ and converge pointwise as $g \rightarrow \infty$ and $s \rightarrow \infty$. Therefore:

$$
\lim _{s \rightarrow \infty} \lim _{g \rightarrow \infty} \Psi_{s, g}\left(\varrho_{s, g}, \beta, \lambda\right)=\lim _{s \rightarrow \infty} \Psi_{s}\left(\varrho_{s}, \beta, \lambda\right)=\Psi(\tilde{\varrho}, \beta, \lambda) .
$$

On the other hand one certainly has:

$$
\min _{\varrho \in \Omega} \Psi(\varrho, \beta, \lambda) \geqq \lim _{s \rightarrow \infty} \lim _{g \rightarrow \infty} \min _{\varrho \in \Omega} \Psi_{s, g}(\varrho, \beta, \lambda),
$$

so that the minimum is attained at $\tilde{\varrho}$ :

$$
\Phi(\beta, \lambda)=\Psi(\tilde{\varrho}, \beta, \lambda)=\lim _{s \rightarrow \infty} \lim _{g \rightarrow \infty} \Phi_{s, g}(\beta, \lambda)=\lim _{N \rightarrow \infty} f(N, \beta, \lambda) .
$$

\section{Taking the Derivative}

In order to be able to apply Theorem A2 of the appendix, we have to make sure that the $\tilde{\varrho}=\tilde{\varrho}(\lambda)$ vary continuously with $\lambda$ at $\lambda=0$. We can use the same considerations for the limit $\lambda \rightarrow 0$ as for $g \rightarrow \infty$ and $s \rightarrow \infty$ : there are converging subsequences $\tilde{\varrho}\left(\lambda_{i}\right) \rightarrow \tilde{\varrho}(0)$ as $\lambda_{i} \rightarrow 0$. This implies continuity, if $\tilde{\varrho}(0)$ is unique. We claim the following

Theorem. If $\beta$ is not the critical value $\beta_{c}$, then the solutions of the T.F.-Equations (18) and (19) in a spherical volume and at $\lambda=0$ are rotation invariant and unique.

Proof. We characterize classes $K_{m}$ of densities $\varrho$ by real-valued functions $m(r)$ :

$$
K_{m}=\left\{\varrho \in \Omega: \int_{V} \theta(r-\varrho(x)) d^{3} x=m(r) \forall r\right\} .
$$


Every term of the form $\int_{V} F\left(\varrho(x) d^{3} x\right.$ is a functional, which depends only on the class $K_{m}$ of $\varrho$. In $\Psi(\varrho, \beta, \lambda)$ only the term $\int_{V \times V} d^{3} x d^{3} y \varrho(x) V(x-y) \varrho(y)$ is not of this form. But this term is minimized by the unique spherically symmetric and decrasing function in $K_{m}$ [6]. (We specify now that $V$ shall have the form of a sphere.) For spherically symmteric functions one knows that the solution of (18), (19) at $\lambda=0$ are unique $\left(\tilde{\varrho}(0)=\varrho_{T F}\right)$ unless the system is at the critical point $\beta_{c}$, where a phasetransition occurs [2].

Q.e.d.

In the last step we use (9), (33), Griffith's Lemma [7] and Theorem A2. The result is:

$$
\begin{aligned}
& \lim _{N \rightarrow \infty}\left\langle\hat{\varrho}_{N}\left(w_{1} \ldots w_{n}\right)\right\rangle_{\beta}=\left.\frac{\partial}{\partial \lambda} f(N, \beta, \lambda)\right|_{\lambda=0}=\left.\frac{\partial}{\partial \lambda} \Phi(\beta, \lambda)\right|_{\lambda=0} \\
& =\left.\frac{\partial}{\partial \lambda} \Psi\left(\varrho_{T F}, \beta, \lambda\right)\right|_{\lambda=0}=\prod_{i=1}^{n} \int \varrho_{T F}(x) w_{i}(x) d^{3} x, \quad \text { if } \quad \beta \neq \beta_{c} .
\end{aligned}
$$

This means that the expectation values of the density converge as tempered distributions to the T.F.-density and are not correlated in the limit. A related result for the T.F.-theory of atoms $([8,9])$ suggests that the densities converge as distributions over the test-function space $L^{5 / 2}(V)$.

\section{Appendix: Two Theorems on Concave Functions}

Theorem A1. Let a concave function $f(\lambda)$ be defined by a variational principle:

$$
f(\lambda)=\min _{\varrho \in \Omega} F(\varrho, \lambda)
$$

and such that the minimum is attained at some $\varrho_{\lambda}$ :

$$
f(\lambda)=F\left(\varrho_{\lambda}, \lambda\right)
$$

Furthermore, we suppose that the partial derivatives

$$
F^{\prime}\left(\varrho, \lambda_{0}\right)=\left.\frac{\partial}{\partial \lambda} F(\varrho, \lambda)\right|_{\lambda=\lambda_{0}}
$$

exist for all pairs $\left(\varrho, \lambda_{0}\right)$. Then the following relation holds:

$$
f_{+}^{\prime}(\lambda) \leqq F^{\prime}\left(\varrho_{\lambda}, \lambda\right) \leqq f_{-}^{\prime}(\lambda) .
$$

$f_{+}^{\prime}\left(f_{-}^{\prime}\right)$ is the right- (left-) hand-side derivative, which exists, since $f$ is concave [10].

$$
\text { Proof. } \begin{aligned}
f_{+}^{\prime}(\lambda)= & \lim _{\varepsilon \rightarrow 0}(1 / \varepsilon)\left[F\left(\varrho_{\lambda+\varepsilon}, \lambda+\varepsilon\right)-F\left(\varrho_{\lambda}, \lambda\right)\right] \leqq \lim _{\varepsilon \rightarrow 0}(1 / \varepsilon)\left[F\left(\varrho_{\lambda}, \lambda+\varepsilon\right)\right. \\
& \left.-F\left(\varrho_{\lambda}, \lambda\right)\right] \\
=F^{\prime}\left(\varrho_{\lambda}, \lambda\right)= & \lim _{\varepsilon \rightarrow 0}(1 / \varepsilon)\left[F\left(\varrho_{\lambda}, \lambda\right)-F\left(\varrho_{\lambda}, \lambda-\varepsilon\right)\right] \\
\leqq & \lim _{\varepsilon \rightarrow 0}(1 / \varepsilon)\left[F\left(\varrho_{\lambda}, \lambda\right)-F\left(\varrho_{\lambda-\varepsilon}, \lambda-\varepsilon\right)\right]=f_{-}^{\prime}(\lambda) .
\end{aligned}
$$


Theorem A2. If it is possible to attach a topology to $\Omega$ such that $F^{\prime}(\varrho, \lambda)$ is a continuous function on $\Omega \times R$ and if there exists a path of minimizing $\varrho_{\lambda}$, continuous at $\lambda_{0}$, then $f(\lambda)$ is differentiable at $\lambda_{0}$ and

$$
f^{\prime}\left(\lambda_{0}\right)=F^{\prime}\left(\varrho_{\lambda_{0}}, \lambda_{0}\right)
$$

Proof. Since $f$ is concave, for $x>y$ the relations ${f^{\prime}}_{+}(x) \leqq f^{\prime}-(x) \leqq f_{+}^{\prime}(y)$ hold. For $\varepsilon>0$ and with Theorem A1:

$$
F^{\prime}\left(\varrho_{\lambda_{0}+\varepsilon}, \lambda_{0}+\varepsilon\right) \leqq f_{-}^{\prime}\left(\lambda_{0}+\varepsilon\right) \leqq F^{\prime}\left(\varrho_{\lambda_{0}}, \lambda_{0}\right) \leqq f_{-}^{\prime}\left(\lambda_{0}\right) \leqq f_{+}^{\prime}\left(\lambda_{0}-\varepsilon\right) \leqq F^{\prime}\left(\varrho_{\lambda_{0}-\varepsilon}, \lambda_{0}-\varepsilon\right) .
$$

The difference between the right- and left-hand-side tends to zero with $\varepsilon \rightarrow 0$ because of the continuity.

Q.e.d.

Acknowledgements. This work has been done as part of a doctoral thesis at the "Institut für theoretische Physik" at the university of Vienna. It is a pleasure to express my gratitude to Prof. W. Thirring, from whom I learned mathematical physics, to my colleagues, and to Prof. P. Hertel for uncountable useful discussions.

\section{References}

1. Hertel, P., Narnhofer, H., Thirring, W.: Commun. math. Phys. 28, 159-167 (1972)

2. Hertel, P., Thirring, W.: Thermodynamic Instability of a System of Gravitating Fermions. In: Dürr,H.P. (Ed.): Quanten und Felder. Braunschweig: Vieweg 1971

3. Thirring, W.: Vorlesungen über mathematische Physik T7: Quantenmechanik. Lecture notes

4. Narnhofer, H., Thirring, W.: Acta Phys. Austr. 41, 281-297 (1975)

5. Maison, H. D.: Commun. math. Phys. 22, 166-172 (1971)

6. Lichtenstein, L.: Math. Z. 3, 8-10 (1918)

7. Griffiths, R. B.: J. Math. Phys. 5, 1215-1222 (1964)

8. Baumgartner,B.: The Thomas-Fermi-Theory as Result of a Strong-Coupling-Limit. To be published

9. Thirring, W.: Vorlesungen über mathematische Physik T8: Quantenmechanik großer Systeme. Lecture notes

10. Roberts, A., Varberg, D.: Convex Functions. London: Academic Press 1973

Communicated by G. Gallavotti

Received November 7, 1975 
